\title{
Scheduling Data-Parallel Computations on Heterogeneous and Time-Shared Environments
}

\author{
Salvatore Orlando ${ }^{1}$ and Raffaele Perego ${ }^{2}$ \\ 1 Dip. di Matematica Appl, od linformatica, Universith̀ Ca' Foscari di Venezia, ltaly \\ ${ }^{2}$ Istituto CNUCE, Consiglio Nazionale delle Ricerche (CNR), Pisa, Italy
}

\begin{abstract}
This paper addresses the problem of load balancing data-parallel computations on heterogeneous and time-shared parallel computing environments, where load imbalance may be introduced by the different capacities of processors populating a computer, or by the sharing of the same computational resources among several users. To solve this problem we propose a run-time support for parallel loops based upon a hybrid (static + dynamic) scheduling strategy. The main features of our teclmique are the absence of centralization and synchronization points, the prefelching of work toward slower processors, and the overlapping of communication latcncies with useful computation.
\end{abstract}

\section{Introduction}

It is widely held that distributed and parallel computing disciplines are converging. Advances in network technology have in fact strongly increased the network bandwidth of state-of the art distributed systems. $\Lambda$ gap still exists with respect to communication latencies, but this difference too is now much less dramatic. Furthermore, most Massively Parallel Systems (MPSs) are now built around the same off-the-shelf superscalar processors that are used in high performance workstations, so that fine--grain parallelism is now exploited intra-processor rather than inter-processor. The convergence of parallol and distribuled computing disciplines is also more evident if we consider the programming models and cnvironments which dominate current parallel programming: MPI, PVM and Iligh Performance Fortran (IIPF) are now available for both MPSs and NOWs.

'This trend has a direct impact on the research carried ont on the two converging disciplines. For example, parallel computing research has to deal with problems introduced by heterogencity in parallel systems. This is a typical feature of distributed systems, but nowadays helerogencous NOWs are incrcasingly used as parallel compuling resources [2], while MJ'Ss may be populated with different of [-the-shelf processors (e.g. a SCI/Cray 'T3E system at the time of this writing may be populated with 300 or $450 \mathrm{MII}$ DEC $\Lambda$ plia processors). Furthermore, some Ml'Ss, which were normally used as batch machines in space sharing, may now be concurrently used by different users as time shared mulliprogrammed enviromments (e.g. an IBM SP2 system can be configured in a way that allows users to specify whether the processors must be acquired as shared or dedicated resources). This paper focuses on one of the main problems programmers thus have to deal with on both parallel and distributed systems: the problem 
of "system" load imbalance due to heterogeneity and time sharing of resources. Here we restrict our vicw to dala-parallel computations expressed by means of parallel loops.

In previous works we consiclered imbalances introduced by non-uniform dataparallel computations to be rum on homogeneous, distributed memory, MIMD parallel systems $[11,9,10]$. We devised a novel compiling technique for parallel loops and a related rin lime support (SUPPI.E). 'This pajer showe how SUPPLE can also be utilized to implement loops in all the cases where load imbalance is not a characteristic of the user code, but is caused by variations in capacities of processing nodes. Note that, much other research has been conducted in the field of run-time supports and compilation methods for irregular problems $[13,12,7]$. In our opinion, besides SUPPLE, many of these techniques can be also adopted when load imbalance derives from the use of a time-shared or heterogeneous parallel system. These techiques should also be compared with those specifically devised to face load imbalance in NOW enviromments [4, 8].

SUPPLE is based upon a hybrid scheduling strategy, which dynamically adjusts the workloaks in the presence of variations of processor capacities. The main features of SUPPLlic are the cllicient implementalion of regutar stencil communications, the hybricl (static + (lynamic) scheduling of clunks of ilcrations, and the exploitation of aggressive chunk prefetching to reduce waiting times by overlapping comununication with useful computation. We report performance results of many experiments carried out on an SGI/Cray T3E and an IBM SP2 system. The synthetic benchmarks used for the experiments allowed us to model difterent situations by varying a few important parameters such as the computational grain and the capacity of each processor. The results obtained suggest that, in the absence of a priori knowledge about the relative capacitics of the processors that will actually exccute the program, the hybrid strategy adopted in SUPPLE yields very good performance.

'The paper is organized as follows. Siection 2 presents the synthetic benchmarks and the inachines used for the experiments. Section 3 describes our run-time support and its load balancing strategy. 'The experimental results are reported and discussed in Section 4, and, finally, the conclusions are drawn in Section 5.

\section{Benchmarks}

We adopted a very simple benchmark program that resembles a very common pattern of parallelism (e.g. solvers for diflerential equations, simulations of physical phenomena, and image processing applications). 'T'he pattern is data-parallel and "regular", and thus considered easy to implement on homogencous and dedicated parallel systems. In the benchmark a bidimensional array is updated itcratively on the basis of the old valucs of its elements, while array data referenced are modeled by a five-point stencil. 'The simple IIPF code illustrating the benchmark is shown below.

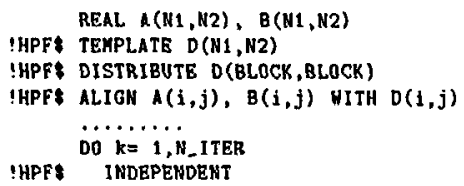




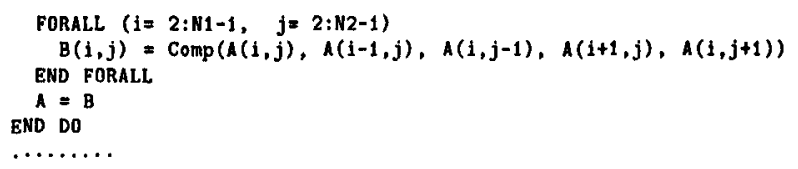

Note the BHLOCK distribution to exploit data locality by reducing off local-memory data references. 'The actual computation performed by the benchmark above is hidden by the function Comp(). We thus prepared several versions of the same benchmark where Comp() was replaced with a dunmy computation characterized by known and fixed costs. Moreover, since it is important to observe the performance of our loop support when we increase the number of processors, for each different grid $P$ of processors we modified the dimensions of the data set to keep the size of the block of data allocated to each processor constant. Finally, another feature that we changed during the tests is N_ITER, the number of iterations of the external sequential loop. 'This was done to simulate the behavior of real applications such as solvers of differential equations, which require the same parallel loops to be cxeculed many times, and image filtering applications, which usually perform the update of the input image in just one step).

\section{The SUPPLE Approach}

SUPPLE (SUPport for Parallel Loop Execution) is a portable run-time support for parallel loops $[9,11,10]$. It is written in $\mathrm{C}$ with calls to the MPI library.

The main innovative feature of SUPPLE [9] is its ability to allow data and computation to be clynamically migrated, without losing the ability to exploit all the static optimizations that can be adopted to eflicicntly implement stencil data references.

Stencil implementation is straightforward, duc to the regularity of the blocking data layout adopted. For each array involved SUPPLE allocates to each processor enough memory to host the block partition, logically subdivided into an inner and a perimeler region, and a surrounding ghost region. The ghost region is used to buffer the parts of the perimeler regions of the adjacent partitions that are owned by neighboring processors, and are accessed through non local references. The inner region contains data elements that can be computed without fetching external data, while to compute data elements belonging to the perimeler region these external data have to be waited for. I,oop iterations are statically assigned to each processor according to the owner compules rulc, but, to overlap communications with useful computalions, iterations are reordered [3]: the iterations that assign data items belonging to the inner region (which refer local data only) are scheduled between the asynchronous sending of the perimeter region to neighboring processors and the receiving of the corresponding daila inlo the ghost region. This static sclochuling may be changed at run-time by migrnting iterations, but, in order to avoid the introduction of irregularities in the implementation of stencil data reference, only iterations updating the inner region can be migrated. We group these iterations into chunks of fixed size $g$, by statically tiling the inner region. SUPPLE migratcs chunks and associated data tiles instead of single iterations.

At the beginning, to reducc overheads, each processor statically executes its chunks, which are stored in a queuc $Q$, hereafter local queue. Once a processor understands that. 
its local queue is becoming empty, it autonomously decides to start the dynamic part of its scheduling policy. It tries to balance the processor workloads by asking overloaded partners for migrating both chunks and corresponding data tiles. Note that, due to stencil data references, to allow the remote execution of a chunk, the associated tile mush be accompanicd by a surronding area, whose size depends on the specific stencil features. Migrated chunks and data tiles are stored by each receiving processor in a qucue $R Q$, called remole. Since load balancing is started by underloaded processors, our techmique can be classified as receiver initiated $[6,14]$. In the following we detail our hybrid scheduling algorithm.

During the initial static phase, each processor only executes local chunks in $Q$ and measures their computational cost. Note that, since the possible load imbalance may only derive from different "speeds" of the processors involved, chunks that will possibly be migrated and stored in $R Q$ will be considered as having the same cost as the ones stored in $Q$. 'Thus, on the basis of the knowledge of the chunk costs, each processor estimates its current load by simply inspecting the size of its queues $Q$ and $R Q$.

When the estimated local load becomes lower than a machine-dependent Threshold, each processor antonomonsly sharis the dymamic part of the scheduling technique and starts asking other processors for remote chunks. Correspondingly, a processor $p_{j}$, which receives a migration request from a processor $p_{i}$, will grant the request by moving some of its workload to $p_{j}$ only if its current load is higher than Threshold. To reduce the overheads which might derive from requests for remote chunks which cannot be served, each processor, when its current load becomes lower than Threshold, broadcasts a socalled termination message. 'Therefore the round-robin strategy used by underloaded processors to choose a partner to be asked for further work skips terminated processors. Once an overloaded processor decides to grant a migration request, it inust choose the most appropriate number of chumks to be migrated. 'To this end, SUPPLE uses a modified Farloring scheme [5], which is a Sclf Scheduling heuristics formerly proposed to address the efficient implementation of parallel loops on shared-menory inultiprocessors

Finally, the policies exploited by SUPPLE to manage data coherence and termination detection are also fully distribuled and asynclıronous. $\Lambda$ full/empty-like technique [1] is used to asynchronously manage the colıcrence of migrated data tiles. When processor $p_{i}$ sencls a chunk $b$ to $p_{j}$, it sets a llag marking the data tiles associated with $b$ as invalid. 'The next time $p_{i}$ needs to access the same tiles, $p_{i}$ checks the flag and, if the flag is still set, waits for the updated clata tiles from node $p_{j}$. $\Lambda$ s far as termination detection is concerned, the rolo of a processor in the parallel loop execution finishes when it has already recrived a temmination message from all the other processors, and bolh its queues $Q$ and $R Q$ are emply.

In summary, unlike other proposals $[12,4]$, the dynamic scheduling policy of SUPPLE is fully distributed and based upon local knowledge about the local workload, and thus there is no need to synchronize the processors in order to exchange updated information about the global workload. Moreover, SUPPLE may also be employed for applications composed of a single parallel loop, such as filters for image processing. Unlike olher proposals $[13,8]$, it does not exploit past knowledge about the work- 
load distribution at previous loop iterations, since dynamic scheduling decisions are asynchronously taken concurrently with useful computation.

\section{Experimental Results}

All experiments were conducled on an IBM SP2 system and an SGI/Cray T3E. Nole that both machines might be heterogeneous, since both can be equipped with processors of cliffcrent capacitics. 'Tlo 'T3E can in fact be popnlaied with DEC Alpha processors of different generations ${ }^{1}$, while IBM cnables choices from three distinct types of nodes - high, wide or thin - where the differences are in the number of per-node processors, the type of processors, the clock rates, the type and size of caches, and the size of the main memory. Ilowever, we used the SI'2 (a 16 node system equipped with $66 \mathrm{MHz}$ lOWWh, 2 wide processors) as a homogencous time-shared environment. 'To simulate load imbalance we simply launched some compute bound processes on a subset of nodes. On the other hand, we used the T3E (a system composed of $64300 \mathrm{MIIz}-\mathrm{DEC}$ Alpha 21164 processors) as a space-shared heterogencous system. Since all the nodes of our system are identical, we had to simmlate the presence of processors of different spects by introducing an extra cost in the computation performed by those processors considered "slow". Thus, if the granularity of $\operatorname{Comp}()$ is $\mu \mu \mathrm{sec}$ (including the time to read and write the data) on the "fast" processors, and $F$ is the factor of slowdown of "he "slow" ones, the granularity of Comp () on the "slow" processors is $(F \cdot \mu) \mu$ sec. "o prove the effectiveness of SUPPLE, we compared each SUPPLE implementation of e benchmark with an equivalent static and optimnized implementation, like the one ploited by a very efficicut IIPF compiler.

We present several curves, all plotting an exccution time ratio (ETR), i.e. the ratio of the time taken by the stalic scheduling version of the benchmark over the time laken by the SUPPLE hybrid scheduling version. IJence, a ratio greater than one corresponds to an improvement in the total exccution time obtained by adopting SUPPLE with respect to the static version. Bach curve is relative to a distinct granularity $\mu$ of $\operatorname{Comp}()$, and plots the F'TRs as a function of the number of processors employed. 'The size of the data set has been nodified according to the number of processors to keep the size of the sub-block stalically assigned to each processor constant.

\subsection{Time-sliared Environments}

First of all, we show the results obtained on the SP2. Due to the difliculty in running probing experiments because of the minpredictability of the workloads, as well as for the cxchusive use of some resources (in particular, resources as the SP2 high-performance switch and/or the processors themselves) by other users, the results in this case are not so exhaustive as those reported for the tests run on the T3E. As previously mentioned, on the SP2 we ran our experiments after the introduction of a synthetic load on a subset of the nodes used. In particular, we launched 4 compute-bound processes on

\footnotetext{
1 The Pittsburgh Supercomputing Center has a ']'3E system with 512 application processors of which half runs at $300 \mathrm{MIIz}$ and half at $450 \mathrm{MIIz}$.
} 


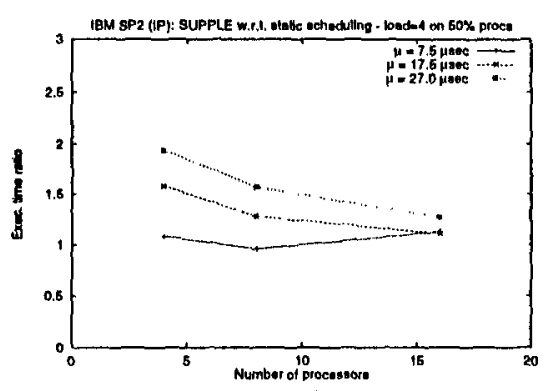

(a)

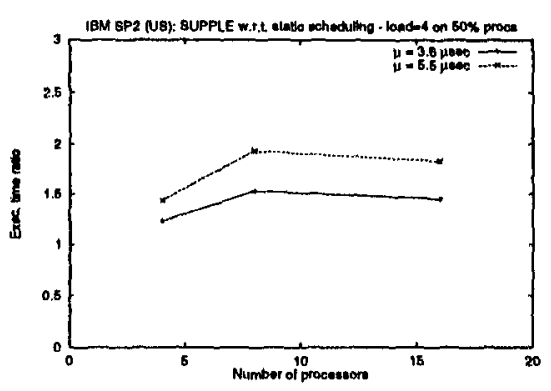

(b)

Fig. 1. SP2 results: ETR results for experiments exploiting (a) the Ethernet, and (b) the high-performance switch

$50 \%$ of the processing nodes employed, while the loads were close to zero on the rest of the nodes. 'This corresponds to "slow" processors characterized by a value of $F$ (factor

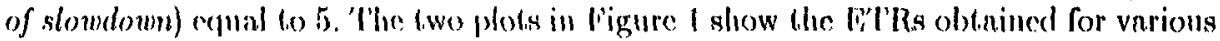
$t h$ and numbers of processors. 'The size of the sub-block owned by cach processor was kept constant and equal to $512 \times 512$, while N_ITER was equal to 5 . As regards the SUPPLE parameters, the Threshold value was set to $0.02 \mathrm{msec}$, and the chunk size $g$ to 32 iterations.

Figure 1.(a) shows the SP2 results obtained by using the Ethernet network and the IP protocol. Note that, even in this case as in the following ones, the performance improvement obtained with SUP'PLE increases in proportion to the size of the grain 11. For $\mu=27 \mu$ sec, and 4 processors, we obtained the best result: SUPPLE implementation is about $100 \%$ raster than the static counterpart. For smaller values of $\mu$, due to the overheads to migrate data, the load imbalance paid by the static version of the benchmark is not large enough to justify the adoption of a dynamic scheduling technique.

Figure 1.(b) shows, on the other liand, the results obtained by running the parallel benchmarks in time-sharing on the SP2 by exploiting the US high-performance switch. Due to the better communication framework, in this case the FTR is favorable to SUP'lle even for smaller values of $\mu$.

\subsection{Heterogeneous Environments}

All the experiments regarding heterogeneous enviromments were conducted on the 'T3E. lleterogeneity was simulated, so that we were able to perform a lot of tests, also experimenting different factors $\vec{F}$ of slowdown.

Iterative benchmark Figure 2 reports the results for the iterative benchmark, where the external sequential loop is repeated 20 times $(\mathrm{N}$ ITER $=20$ ). For all the tests we kept fixed the size of the sub-block assigned to cach processor $(512 \times 512)$. Figures 2.(a) and 2.(b) are relative to an cnviromment where only $25 \%$ of all processors are "slow". 


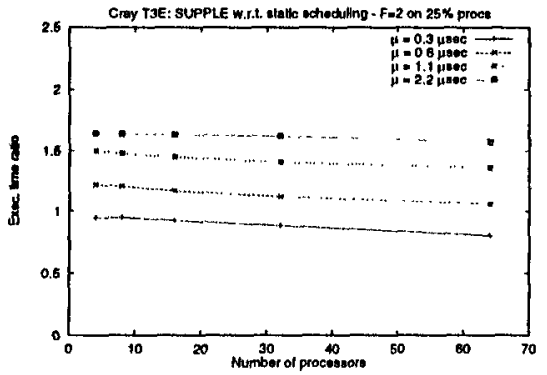

(a)

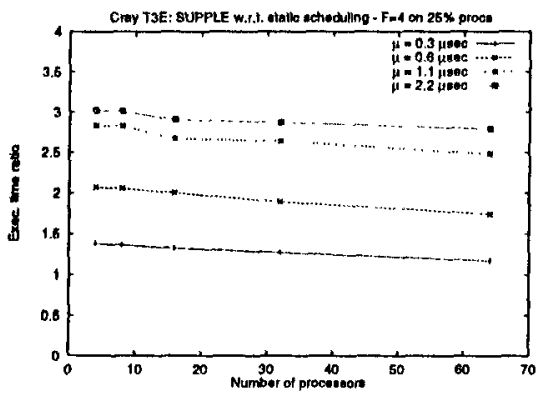

(b)

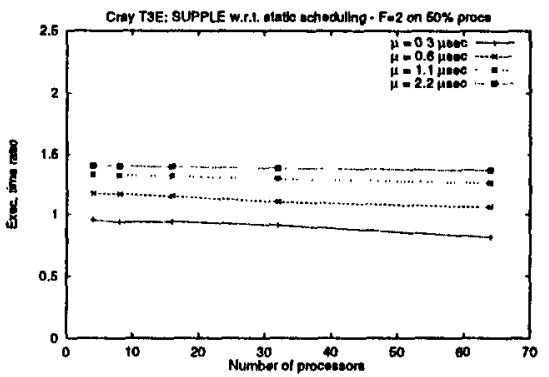

(c)

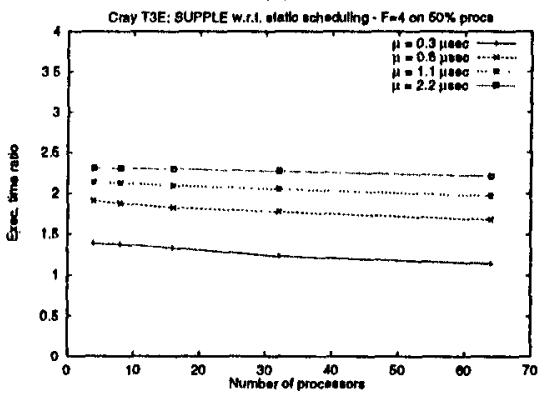

(d)

Fig. 2. T3E results: ETR results for various values of $F$ and $\mu$

'The factors $F$ of slowdown of these processors are 2 and 4, respectively. Figures 2.(c) and 2.(d) refer to an environinent where half of the processors employed are "slow'.

Each curve in all these figures plots the ETR for a benchmark characterized by a distinct $\mu$. With respect to the SP2 tests, in this case we were able to reduce $\mu$ ( $\mu$ now ranges between 0.3 and $2.2 \mu \mathrm{sec}$ ), always obtaining an encouraging performance with our SUPPLE support. Note that such grains are actually very small: for $\mu$ equal to $0.3 /$ sec, about $85 \%$ of the total time is spent on memory accesses (to compute addresses and access dat,a element covering the five-point stencil), and only $15 \%$ on arithmetic operations. We belicve that the cncournging results obtained are due to the smaller overheads and latencies of the T3E network, as well as to the absence of time-sharing of the processor, thus spccding up the responsiveness of "slow" processors to requests coming from "fast." oncs. "Woe reductions in gramularily made it, possible to enlarge the chumk size $g(g=128)$ withont losing the effectiveness of the dymamic scheduling algorithm. 'The threshold ranged between 0.02 and $0.06 \mathrm{msec}$, where larger values were used for larger $\mu$.

Looking at Figure 2, we can see that better results (SUPPLE execution times up to 3 times lower than those obtained with static scheduling) were obtained when the "slow" processors were only $25 \%$ of the total number. The reason for this behavior is clear: in this casc, we have more "fast" processors to which the extra workload previously assigned to the "slow" processors can be dynanically distributed. 'T'he execution times 
obtained with the static implementation are, on the other hand, almost independent of the percentage of "slow" processors. In fact, even if only one of the processors was "slow", its execution time would dominate the overall completion time.

We also tested SUPPLE on a homogencous system (i.e. a balanced one) in order to cvaluate its overhend w.r.t. a static implementation, which, in this case, is optimal. 'The overhead is almost constant, for data sets of the same sizes subdivided into a given number of chunks, but its iufluence becomes larger for smaller granularities because of the shorter execution time and the limited possibility of hiding communication latencies with computations. Thus, for $\mu=2.2$ /sec the two execution times are almost comparable, while for $\mu=0.3 \mu \mathrm{sec}$, the static version of the benchmark becomes $60 \%$ faster than SUPPLE. We verified that the overhead introduced by SUPPLE is due to some undesired migration of chunks, and to the dynamic scheduling technique which entails polling the network interface to check for incoming messages (even if these messages do not actually arrive).

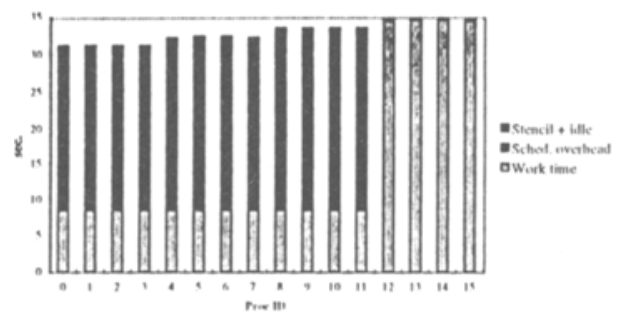

(a)

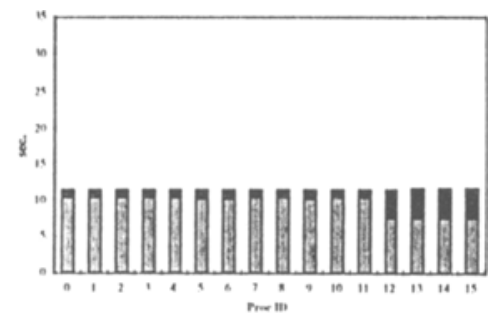

(b)

Fig. 3. Work time and overhcads for a static (a) and a SUPPLE (b) version of the benchmark

Finally, we instrumented the static and the SUPPLE versions of a specific benchmark to evaluate the ratio between the execution time spent on useful computations and on dynamic scheduling overheads. 'I'he features of the benchmark used in this case are the following: a $2048 \times 2048$ dalia set distributed over a grid of $4 \times 4$ T3E processors, and an external sequential loop iterated for 20 times. The sinulated unbalanced enviroument was characterized by $25 \%$ of "slow" processors, with a slowdown factor of 4. Pigure 3.(a) sliow the results oblained by rumning the static implementation of this benchmark. It is worth noting the work time on the "slow" processors, which is 4 limes the work time on the "fast" ones. 'The black portions of the bars show idle times on "fast" processors while waiting for border dala. 'l'hese idle limes are thus due to communications implementing stencil data references, where corresponding send/receive on fast/slow processors are not synchronized due to their different capacities.

Figure 3.(b), on the other hand, shows the SUPPLE results on the same benchmark. Note the redistribution of workloads from "slow" to "fast" processors. Thanks to dynamic load balancing, idle times disappeared, but we have larger scheduling overheads due to the communications used to dynamically migrate chunks. These overheads are 
clearly larger on "slow" processors, which spend a substantial part of their execution tine on giving away work and on receiving the results of migrated iterations.

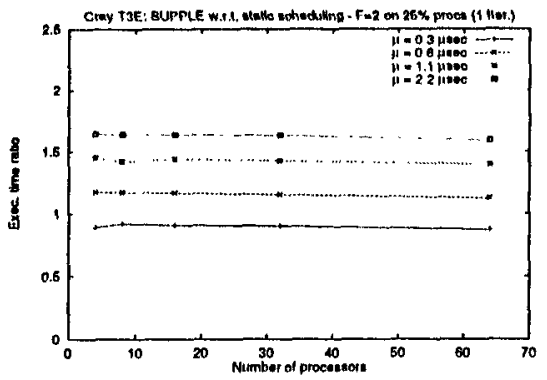

(a)

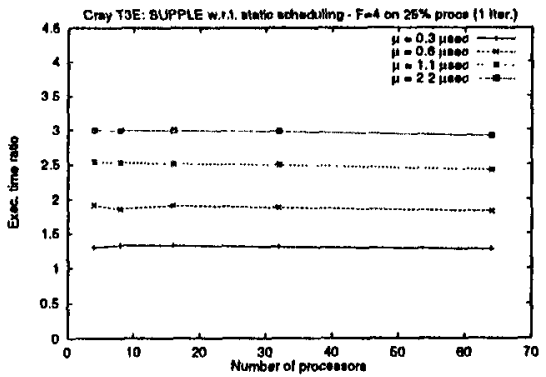

(b)

Fig. 4. T3E results: E'I'll results oblained ruming a single iteration benchmark for varions values of $F$ and $\mu$

Single iteration benchmark $\Lambda$ s explained above, one of the advantages of SUPPLE is that it can also be used for balancing parallel loops that have only to be executed once. In this case some overheads cannot be overlapped, and at the end of loop execution "slow" processors have to wait for the results of iterations executed remotely. Figure 4 shows the encouraging EIRs obtained by our SUPPLE implementation w.r.t. the static one. Note that all the results plotted in the figure refer to unbalanced environments where only $25 \%$ of the processors are "slow". Moreover, due to the larger data sets used for these tests, the FIIts are in some cases even more favorable for SUPPLE than the ones for iterative benchmarks.

\section{Conclusions}

We have discussed the implementation on heterogeneous and/or timed-shared parallel machines of regular and uniform parallel loop kernels, with statically predictable stencil data references. We have assumed that no information about the capacities of the processors involved is available until run-time, and that, in time-shared environments, these capacities may change during run time. 'To implement the kernel benchmark we cmployed SUPPIS, a mu-lime support the we hat previously introduced to compile non-uniform loops.

The tests were conducted on an SGI/Cray T3E and an IBM SP2. We compared the SUPPLE results with a static implementation of the benchmark, where data and computations are evenly distributed to the various processing nodes. The SP2, a parallel system that can be used as a time-shared NOWs, was loaded with artificial computebound processes before running the tests. On the other hand, we needed to simulate a heterogencous SGI/Cray 'MSI', i.c. a machine whose nodes may be equipped with 
different off-the-shelf processors and/or memory organization. The performance results were very encouraging. On the SP2, where half of the processors were loaded with 4 compute-bound processes, the SUPPLE version of the benchmark resulted at most $100 \%$ faster than the stalic onc. On the T3E, clepending on the amount of "slow" processors, on the mumber of processors employed and the granularity of loop itcrations, the SUPPIJ version reached percentages of performance improvement ranging between $20 \%$ and $270 \%$.

Further work has to be done to compare our solution with other dynamic scheduling strategies proposed elsewhere. More exhaustive experiments with different benchmarks and dymanic variations of the system loads are also required to fully evaluate the proposal. Ilowever, we believe that hybrid strategies like the one adopted by SUPPLE can be profitably exploited in many cases where locality exploitation and load balance must be solved at the same time. Morcover, our strategy can be easily integrated in the compilation model of a high level data parallel language.

\section{References}

I. R. Alverson el al. The Tera computer system. In Proc. of the 1990 ACM Int. Conf. on Supercomputing, pages 1-6, 1990.

2. A. Anurag, G. Edjlali, and J. Saltz. The Utility of Exploiting Idle Workstations for Parallel Computation. In Proc. of the 1997 ACM SIGMETRICS, July 1997.

3. S. Hiranandani, K. Kennedy, and C. 'Tseng. Evaluating Compiler Optimizations for Fortran D. J. of Parallel and Distr. Comp., 21(1):27-45, April 1994.

4. S. Ilym Ilummel, J. Schmidt, R. N. Uma, and J. Wein. Load-Sharing in Heterogeneous Systems via Weiglited Jactoring. In Proc. of the 8th SPAA, July 1997.

5. S.F. Hummel, E. Schonberg, and L.E. Flym. Factoring: $\Lambda$ Method for Scheduling Parallel Loops. Cormm. of the ACM, 35(8):90-101, Aug. 1992.

6. V. Kumar, A.Y. Grama, and N. Rao Vempaty. Scalable Load Balancing 'Techniques for Parallel Computers. J. of Parallel and Distr. Comp., 22:60-79, 1994.

7. J. Liu and V. A. Salctore. Self-Scheduling on Distributed-Memory Machines. In Proc. of Supercomputing '93, pages 814-823, 1993.

8. M. Colajanni M. Cermele and G. Necci. Dynamic Load Balancing of Distributed SPMD Computations with Explicit Message-Passing. In Proc. of the IEEE Workshop on Heterogeneous Computing, pages 2-16, 1997.

9. S. Orlando and R. Perego. SUPl'te: an Bificient Rem-Time Support for Non-Uniform Parallel Loops. 'Jechnical Report 'J'R-17/96, Dipartimento di Mat. Appl. ed Informatica, Università di Venezia, Dec. 1996. 'l'o appear on J. of System Architecture.

10. S. Orlando and R. Perego. $\Lambda$ Comparison of lmplementation Strategies for Non-Uniform Data Parallel Computations. Tecluical Report. 'TR-9/97, Dipartimento di Mat. Appl. ed Informatica, Universitè di Venczia, $\Lambda$ pril 1997. Under revision for publication on the J. of Parallel and Distr. Comp.

11. S. Orlando and R. Perego. A Support for Non-Uniform Parallel Loops and its Application to a Flame Simulation Code. In Proc. of the $4^{\text {th }}$ Int. Symposium, IRREGULAR '97, pages 186-197, Paderborn, Germany, June 1997. LNCS 1253, Spinger-Verlag.

12. O. Plata and F. F. Rivera. Combining static and dynamic scheduling on distributedmemory multiprocessors. In Proc. of the 1994 ACM Int. Conf. on Supercomputing, pages $186-195,1994$. 
13. J. Saltz et al. Runtime and Language Support for Compiling Adaptive Irregular Programs on Distributed Memory Machines. Software Practice and Experience, 25(6):597-621, June 1995.

14. M.II. Willebeek-LeMair and A.P. Reeves. Strategies for Dynamic Load Balancing on Highly Parallel Computers. IEEE Trans. on Parallel and Distr. Systems, 4(9):979-993, Scpt. 1903, 